\title{
Acid-Base Cosolvent Method for Determining Aqueous Permeability of Amiodarone, Itraconazole, Tamoxifen, Terfenadine and Other Very Insoluble Molecules ${ }^{1)}$
}

\author{
Jeffrey Alan Ruell, Oksana Tsinman, and Alex AvdeEF* \\ pION INC; 5 Constitution Way, Woburn, MA 01801-1024, U.S.A. \\ Received December 1, 2003; accepted February 16, 2004
}

A high-throughput, UV-detection PAMPA (parallel artificial membrane permeability assay) cosolvent procedure is described, based on the use of $20 \% \mathrm{v} / \mathrm{v}$ acetonitrile in aqueous buffer. A training set of 32 drugs (17 bases, 13 acids, 2 ampholytes) was studied both in aqueous buffer and in cosolvent-buffer solutions. A procedure was devised, where intrinsic permeability values, $\log P_{0} \cos$, measured in cosolvent solution, are converted to values expected under cosolvent-free conditions, using an in silico model based on Abraham H-bond acidity $(\alpha)$ and basicity $(\beta)$ descriptors, developed with the Algorithm Builder computer program, to obtain aqueous intrinsic permeability values: $\log P_{0}=0.738+0.885 \log P_{0}{ }^{\operatorname{Cos}}-1.262 \alpha+0.436 \beta, r^{2}=0.97, q^{2}=0.96, s=0.38, n=32, F=279$. Five sparingly-soluble weak bases (solubility $<1 \mu \mathrm{g} / \mathrm{ml}$ ), which could not be characterized without cosolvent, had their aqueous intrinsic permeability, $P_{0}$, estimated: miconazole $0.32 \mathrm{~cm} / \mathrm{s}$; itraconazole $3.2 \mathrm{~cm} / \mathrm{s}$; amiodarone $13 \mathrm{~cm} / \mathrm{s}$; tamoxifen $28 \mathrm{~cm} / \mathrm{s}$; terfenadine $162 \mathrm{~cm} / \mathrm{s}$.

Key words PAMPA; Double-Sink; low solubility; permeability

PAMPA (parallel artificial membrane permeability assay), introduced by Kansy et $a l^{2}{ }^{2}$ to predict the oral absorption of new therapeutic agents, has undergone substantial recent development, ${ }^{3-15)}$ and is gaining acceptance in pharmaceutical research. ${ }^{16-19)}$ However, test compounds with very low aqueous solubility $(<20 \mu \mathrm{g} / \mathrm{ml})$ have been very difficult to assay with UV detection. Using other detection systems, such as $\mathrm{LC} / \mathrm{MS}{ }^{3)}$ greatly lowers the assay throughput, and increases its cost. Cosolvents, ${ }^{5)}$ bile salts, ${ }^{20)}$ and other solubilizing agents ${ }^{13)}$ have been tried to improve the permeability assay of low-solubility compounds. We describe here a new general method based on the use of a cosolvent and direct UV detection in a microtitre plate format suitable for cost-sparing high-throughput applications. The new method was used to estimate the aqueous permeability of several sparingly-soluble molecules (intrinsic solubility less than $0.1 \mu \mathrm{g} / \mathrm{ml}$ ), including amiodarone $\left(0.006 \mu \mathrm{g} / \mathrm{ml}^{19)}\right)$, itraconazole $(<0.1 \mu \mathrm{g} /$ $\left.\mathrm{ml}^{19)}\right)$, tamoxifen $\left(0.02 \mu \mathrm{g} / \mathrm{ml}^{19)}\right)$, and terfenadine $(0.095 \mu \mathrm{g} /$ $\mathrm{ml}^{19)}$ ). This was done by performing the assay in $20 \% \mathrm{v} / \mathrm{v}$ acetonitrile/water, and deriving the results corresponding to zero-cosolvent by using a prediction scheme based on Abraham solvation descriptors. ${ }^{21)}$ Our primary objective in the study was to derive the aqueous intrinsic permeability of such problematic molecules.

\section{Experimental}

Chemicals Thirty-eight compounds ( 23 bases, 13 acids, 2 ampholytes) used in the study (Table 1) were purchased from Sigma-Aldrich (St. Louis, MO, U.S.A.), and used as received. PAMPA lipid was obtained from $p \mathrm{ION}$ (Double-Sink ${ }^{\mathrm{TM}}$ lipid, P/N 110669). Spectroscopic grade dimethylsulfoxide (DMSO) was purchased from Burdick \& Jackson (Muskegon, MI, U.S.A.), and was used to prepare fresh $10 \mathrm{~mm}$ sample stock solutions. The $\mathrm{pH}$ of the assayed solutions was adjusted using universal buffers ( $p$ ION, P/N 100621 for $0 \%$ cosolvent and $\mathrm{P} / \mathrm{N} 110158$ for $20 \% \mathrm{v} / \mathrm{v}$ acetonitrile), and with a buffer solution at $\mathrm{pH} 7.4$ containing a surfactant ${ }^{19)}$ to simulate the effect of serum proteins ( $p$ ION Double-Sink ${ }^{\mathrm{TM}}$ ASB-7.4 buffer, P/N 110139).

PAMPA Method The PAMPA Evolution instrument from $p$ ION was used. PAMPA "sandwiches" were formed from a donor 96-well microtitre plate ( $p \mathrm{ION}, \mathrm{P} / \mathrm{N}$ 100611) and a matching filter plate (IPVH-brand from Millipore Corp., Bedford, MA, U.S.A.), coated with a $20 \%$ (w/v) dodecane solution of a lecithin mixture ( $p$ ION P/N 110669). The donor $(50 \mu \mathrm{M} \mathrm{sam}-$ ple) aqueous or cosolvent buffer solutions were varied in $\mathrm{pH}$, while the cosolvent-free acceptor solution had the same $\mathrm{pH}$ 7.4. The $p \mathrm{ION}$ Gut Box ${ }^{\mathrm{TM}}$ $(\mathrm{P} / \mathrm{N} 110205)$ was used to effect individual-well magnetic stirring (180$600 \mathrm{rpm}$ ). The plate sandwich was formed and allowed to incubate in the Gut Box at $25 \pm 1^{\circ} \mathrm{C}$ for $10-20 \mathrm{~min}$ in an atmosphere saturated in humidity, and scrubbed free of oxygen and carbon dioxide. Afterwards, sample concentrations in both the acceptor and donor wells were determined by directUV plate spectrophotometry. ${ }^{19)}$ Permeability coefficients were calculated by taking into account filter porosity and sample mass balance. ${ }^{14,15)}$

$\mathrm{p} K_{\mathrm{a}}^{\text {flux }}$ Method of Determining Intrinsic Permeability Coefficients The permeability barrier is assumed to consist of the artificial lipid membrane (held in place by the microfilter) and the two unstirred water layers (UWL) on each side of the barrier, separating the membrane from the bulk aqueous solution. ${ }^{14)}$ Since serial resistance is additive and permeability is the inverse of resistance,

$$
1 / P_{\mathrm{e}}=1 / P_{\mathrm{u}}+1 / P_{\mathrm{m}}
$$

where $P_{\mathrm{m}}$ is the permeability of the artificial membrane, and $P_{\mathrm{u}}$ is the total UWL permeability coefficient.

For ionizable molecules, the membrane permeability, $P_{\mathrm{m}}$, depends on $\mathrm{pH}$ of the bulk aqueous solution. For monoprotic weak acids and bases, the relationship between $P_{\mathrm{m}}$ and $P_{\mathrm{o}}$, the intrinsic permeability of the uncharged species, may be stated as

$$
P_{\mathrm{m}}=P_{\mathrm{o}} /\left(10^{ \pm\left(\mathrm{pH}-\mathrm{p} K_{\mathrm{a}}\right)}+1\right)
$$

with ' + ' used for acids, and ' - ' used for bases. Combining Eqs. (1) and (2), and converting the result to logarithmic form, produces for monoprotic ionizable molecules

$$
\log P_{\mathrm{e}}=\log P_{\mathrm{e}}^{\max }-\log \left(10^{ \pm\left(\mathrm{pH}-\mathrm{p} K_{\mathrm{a}}^{\text {hux }}\right)}+1\right)
$$

The maximum possible effective (measured) permeability, $P_{\mathrm{e}}{ }^{\max }$, is defined as $\log P_{\mathrm{e}} \mathrm{e}^{\max }=\log P_{\mathrm{u}}-\log \left(1+P_{\mathrm{u}} / P_{\mathrm{o}}\right)$. When $P_{\mathrm{o}}>>P_{\mathrm{u}}$ (low-solubility molecules), $P_{\mathrm{e}}^{\max } \approx P_{\mathrm{u}}$. The "flux" ionization constant, $\mathrm{p} K_{\mathrm{a}}^{\text {flux }}$, refers to the $\mathrm{pH}$ value where the resistance to transport across a permeation barrier is $50 \%$ due to the UWL and $50 \%$ due to the membrane. ${ }^{19)}$ The unknown parameters in Eq. $3, \mathrm{p} K_{\mathrm{a}}^{\text {flux }}, P_{\mathrm{u}}$ and $P_{\mathrm{o}}$, were determined by a weighted nonlinear refinement procedure, with supplied aqueous $\mathrm{p} K_{\mathrm{a}}$ values. ${ }^{14,15,19,22,23)}$ Both cosolvent and cosolvent-free parameters were determined by the same procedure.

Prediction Development Software The derivation of the aqueous intrinsic permeability in silico model from the cosolvent-based permeability values involved the testing of the consequences of applying several soluteproperty descriptors, such as octanol-water partition coefficients, $\log P_{\text {oct }}$, McGowan volume, polar surface area, hydrogen bonding, and so on. The Algorithm Builder (Version 1.6) software program from Pharma Algorithms, Inc. (Toronto, Canada), www.ap-algorithms.com, was used. The models were tested by the multiple linear regression approach, and the final model 
was validated using the "leave-one-out" (LOO) calculation formalism.

\section{Results and Discussion}

Intrinsic Permeability Using the $\mathbf{p} K_{\mathrm{a}}^{\text {flux }}$ Method Figure 1 shows measured $\log P_{\mathrm{e}}^{\mathrm{COS}}-\mathrm{pH}$ data of 12 of the 38 molecules characterized in cosolvent solution. The best-fit of Eq. 3 to the data are represented by the solid curves, and the derived $\log P_{\mathrm{m}}^{\mathrm{COS}}-\mathrm{pH}$ curves, according to Eq. 2, are represented by dashed curves. The dotted lines correspond to the $\log P_{\mathrm{u}}$ values, based on Eq. 3. (The effect of the cosolvent on the values of $P_{\mathrm{u}}$ is very small, well below the experimental errors.) The maximum point in the $\log P_{\mathrm{m}}{ }^{\mathrm{COS}}$ curves corresponds to the intrinsic permeability coefficient, $\log P_{\mathrm{o}}^{\mathrm{COS}}$. This value characterizes the transport of the neutral form of an ionizable molecule in the mixed-solvent solution. In Fig. 1 , the intersections of the horizontal and the diagonal tangents occur at $\mathrm{pH}$ values corresponding to the $\mathrm{p} K_{\mathrm{a}}$ in the dashed curves and $\mathrm{p} K_{\mathrm{a}}^{\text {flux }}$ in the solid curves. The difference between the two $\mathrm{p} K$ a values corresponds to the difference between $\log P_{\mathrm{u}}$ and $\log P_{\mathrm{o}}^{\mathrm{COS}}$.
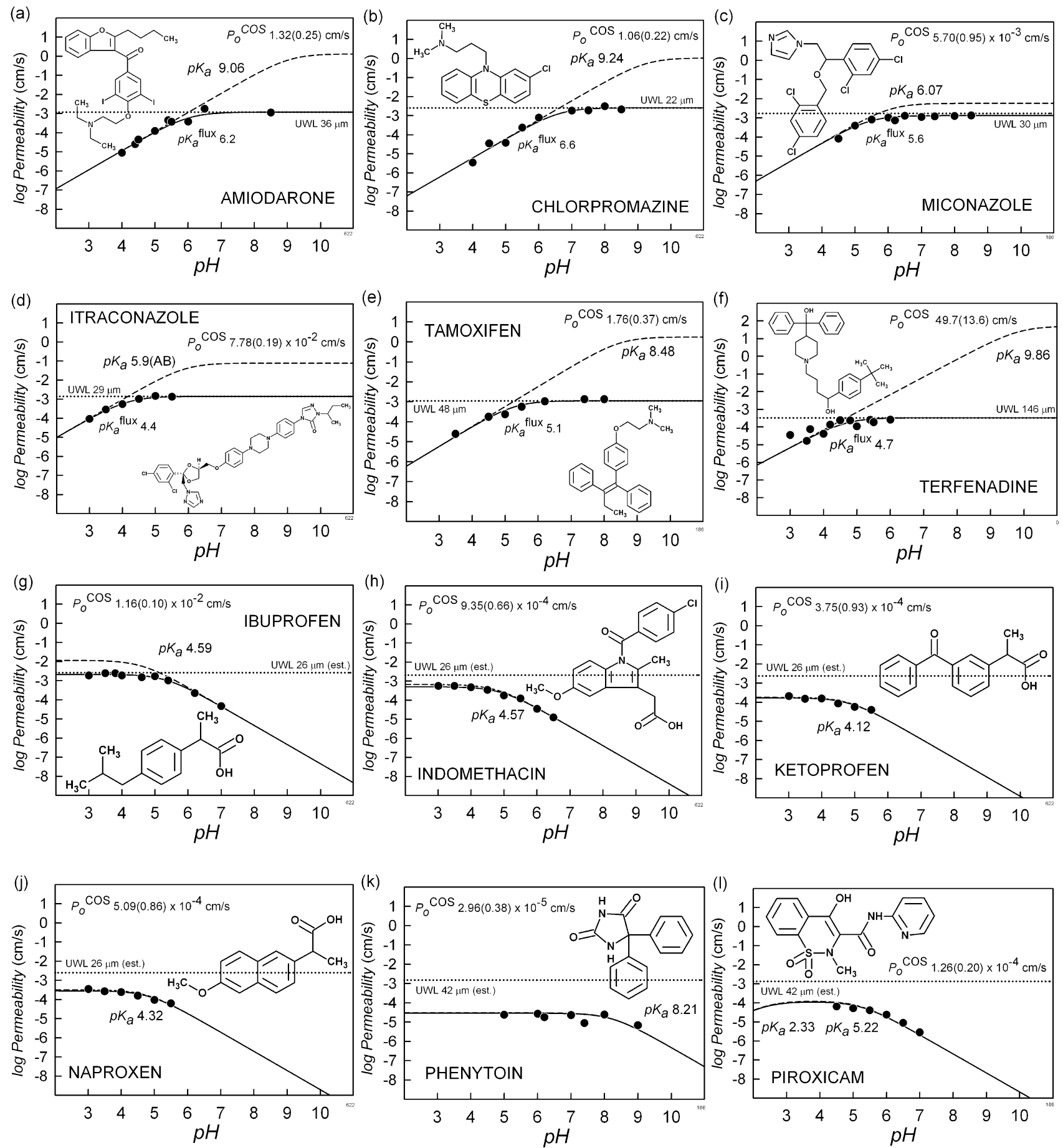

Fig. 1. Log Permeability (cm/s Units) vs. pH Plots for Six Bases and Six Acids

The solid curves represent the best-fit of measured $\log P_{\mathrm{e}}^{\cos } v s . \mathrm{pH}$, according to Eq. 3 . The horizontal tops of the solid curves are approximately (not exactly) equal to log $P_{\mathrm{u}}$. The estimated thicknesses of the UWL are indicated in $\mu \mathrm{m}$ units. The dashed curves are the calculated membrane permeability curves, using Eq. 2 . The horizontal tops of the dashed curves correspond to $\log P_{\mathrm{o}}{ }^{\operatorname{Cos}}$ values. The refined $P_{\mathrm{o}}{ }^{\operatorname{Cos}}$ along with the estimated standard deviations in parentheses are listed in the frames. 
Table 1. Aqueous Instrinsic Permeability Calculated from Cosolvent Intrinsic Permeability ${ }^{a)}$

\begin{tabular}{|c|c|c|c|c|c|c|c|c|c|c|c|}
\hline Compound & Type & MW & $\mathrm{p} K_{\mathrm{a}}$ & & $\log P_{\text {oct }}$ & $\alpha$ & $\beta$ & $\log P_{\mathrm{o}}^{\cos }$ & $\begin{array}{c}\text { Obs } \\
\log P_{\mathrm{o}}\end{array}$ & $\begin{array}{l}\text { Calc } \\
\log P_{\mathrm{o}}\end{array}$ & $\mathrm{Obs}-\mathrm{Calc}$ \\
\hline 2-Naphthoic acid & A & 172.2 & 4.31 & & $3.29^{b)}$ & 0.58 & 0.51 & -3.18 & -2.72 & -2.58 & -0.14 \\
\hline Alprenolol & $\mathrm{B}$ & 249.4 & 9.51 & & 2.99 & 0.61 & 1.11 & -1.05 & 0.02 & -0.47 & 0.49 \\
\hline Chlorpromazine & $\mathrm{B}$ & 318.9 & 9.24 & & 5.40 & 0.00 & 0.87 & 0.03 & 1.62 & 1.15 & 0.48 \\
\hline Doxepin & B & 279.4 & $9.45^{b)}$ & & $4.97^{b)}$ & 0.00 & 1.12 & -0.38 & 0.44 & 0.89 & -0.45 \\
\hline Ergonovine & $\mathrm{B}$ & 325.4 & 6.91 & & 1.67 & 1.26 & 1.71 & -5.17 & -4.14 & -4.67 & 0.54 \\
\hline Flufenamic acid & A & 281.2 & 4.24 & & 5.56 & 0.58 & 0.90 & -1.86 & -1.21 & -1.25 & 0.03 \\
\hline Flumequine & A & 261.5 & 6.59 & & 0.97 & 0.82 & 0.61 & -4.38 & -3.85 & -3.91 & 0.06 \\
\hline Flurbiprofen & A & 244.3 & 4.18 & & 3.99 & 0.61 & 0.65 & -2.46 & -1.78 & -1.93 & 0.16 \\
\hline Gemfibrozil & A & 250.0 & 4.70 & & 3.90 & 0.60 & 0.81 & -1.41 & -1.59 & -0.92 & -0.67 \\
\hline Ibuprofen & A & 206.3 & 4.59 & & 4.13 & 0.63 & 0.62 & -1.94 & -2.11 & -1.50 & -0.62 \\
\hline Indomethacin & A & 357.8 & 4.57 & & 3.51 & 0.57 & 1.20 & -2.88 & -1.65 & -2.01 & 0.36 \\
\hline Ketoprofen & A & 254.3 & 4.12 & & 3.16 & 0.61 & 1.03 & -3.17 & -2.67 & -2.39 & -0.28 \\
\hline Maprotiline & $\mathrm{B}$ & 277.4 & $10.35^{b)}$ & & $5.10^{b)}$ & 0.10 & 0.83 & 1.54 & 2.12 & 2.37 & -0.25 \\
\hline Metoprolol & $\mathrm{B}$ & 267.4 & 9.56 & & 1.95 & 0.61 & 1.50 & -2.15 & -1.17 & -1.27 & 0.10 \\
\hline Morphine & $\mathrm{X}$ & 285.3 & 8.46 & 8.13 & 0.90 & 0.73 & 1.16 & -4.06 & -3.59 & -3.27 & -0.32 \\
\hline Naproxen & A & 230.3 & 4.32 & & 3.24 & 0.61 & 0.88 & -3.12 & -2.30 & -2.41 & 0.10 \\
\hline Ondansetron & B & 293.4 & 7.62 & & 1.94 & 0.00 & 0.76 & -4.26 & -2.38 & -2.70 & 0.32 \\
\hline Penbutolol & $\mathrm{B}$ & 291.4 & 9.92 & & 4.62 & 0.59 & 1.13 & 1.38 & 1.70 & 1.72 & -0.02 \\
\hline Phenazopyridine & $\mathrm{B}$ & 213.2 & 5.15 & & 3.31 & 0.47 & 0.59 & -3.58 & -2.66 & -2.77 & 0.11 \\
\hline Phenytoin & A & 252.3 & 8.36 & & 2.24 & 0.61 & 1.49 & -4.79 & -4.19 & -3.62 & -0.58 \\
\hline Piroxicam & $\mathrm{X}$ & 331.4 & 5.22 & 2.33 & 1.98 & 1.06 & 2.04 & -3.90 & -3.32 & -3.17 & -0.15 \\
\hline Prazosin & $\mathrm{B}$ & 383.4 & 7.11 & & 2.16 & 0.24 & 1.71 & -4.71 & -2.89 & -2.98 & 0.10 \\
\hline Primaquine & $\mathrm{B}$ & 259.4 & 10.03 & 3.55 & 3.00 & 0.17 & 1.32 & -1.22 & 0.56 & 0.02 & 0.54 \\
\hline Probenecid & A & 285.4 & 3.16 & & 3.70 & 0.70 & 1.04 & -2.55 & -1.83 & -1.94 & 0.12 \\
\hline Promethazine & $\mathrm{B}$ & 284.4 & 9.00 & & 4.05 & 0.00 & 1.07 & -0.81 & 0.96 & 0.49 & 0.47 \\
\hline Propranolol & $\mathrm{B}$ & 259.3 & 9.53 & & 3.48 & 0.62 & 1.14 & -0.33 & 0.43 & 0.16 & 0.27 \\
\hline Protriptyline & $\mathrm{B}$ & 263.4 & $10.37^{b)}$ & & $4.91^{b)}$ & 0.09 & 0.79 & 1.36 & 2.43 & 2.17 & 0.26 \\
\hline Quinidine & B & 324.4 & 8.55 & 4.09 & $3.44^{c)}$ & 0.37 & 1.58 & -1.99 & -1.56 & -0.80 & -0.75 \\
\hline Sulphasalazine & A & 398.4 & 8.25 & 2.80 & 3.61 & 1.62 & 1.41 & -4.41 & -4.44 & -4.60 & 0.16 \\
\hline Trimipramine & $\mathrm{B}$ & 294.4 & $9.40^{b)}$ & & $4.84^{b)}$ & 0.00 & 1.16 & 0.74 & 1.58 & 1.90 & -0.32 \\
\hline Verapamil & B & 454.6 & 9.07 & & 4.33 & 0.00 & 1.98 & -1.51 & 0.26 & 0.26 & 0.00 \\
\hline Warfarin & A & 308.3 & 4.97 & & 3.54 & 0.52 & 1.28 & -3.52 & -2.59 & -2.48 & -0.11 \\
\hline Amiodarone & $\mathrm{B}$ & 643.3 & 9.06 & & 7.80 & 0.00 & 0.63 & 0.12 & $\mathrm{nd}^{d)}$ & 1.12 & \\
\hline Itraconazole & $\mathrm{B}$ & 705.6 & $5.90^{b)}$ & & 3.27 & 0.00 & 1.61 & -1.06 & nd & 0.50 & \\
\hline Miconazole & $\mathrm{B}$ & 416.1 & 6.07 & & 4.89 & 0.03 & 1.07 & -1.88 & nd & -0.50 & \\
\hline Tamoxifen & B & 371.5 & 8.48 & & 5.26 & 0.00 & 1.08 & 0.27 & nd & 1.45 & \\
\hline Terfenadine & B & 471.7 & 9.86 & & 5.52 & 0.65 & 1.83 & 1.70 & nd & 2.21 & \\
\hline
\end{tabular}

a) Type: $\mathrm{A}=$ acid, $\mathrm{B}=$ base, $\mathrm{X}=$ ampholyte. $\alpha, \beta=$ Abraham's H-bond acidity, basicity, respectively, as calculated with Algorithm Builder (AB) computer program. The difference between measured and calculated aqueous intrinsic permeability, $\log P_{\mathrm{o}}$, is indicated as obs - calc. The $20 \% \mathrm{v} / \mathrm{v}$ acetonitrile-aqueous intrinsic permeability is indicated as $\log P_{\mathrm{o}}^{\mathrm{COS}}$. All values of $\mathrm{p} K_{\mathrm{a}}$ and $\log P_{\text {oct }}$ were taken from literature compilations ${ }^{19)}$ or were calculated with AB. b) Calculated by AB. $c$ ) Ref. 20 . $d$ ) Not determined, due to very low aqueous solubility of compound.

Table 1 lists the results of the $\mathrm{p} K_{\mathrm{a}}^{\text {flux }}$ method, applied to the training set of 32 ionizable molecules, for both the cosolvent and cosolvent-free aqueous solutions. Five additional molecules (the "application set") at the end of the table are compounds which could only be characterized in cosolvent solution, since these compounds are too low in solubility to be characterized in cosolvent-free buffer. The molecules used in this study are sufficiently lipophilic such that all have intrinsic permeability coefficients nearly equal to or greater than the unstirred water layer permeability, which is a requirement of the $\mathrm{p} K_{\mathrm{a}}^{\text {flux }}$ method. ${ }^{14)}$

Figure 2 shows the training relationship between the aqueous $\log P_{\mathrm{o}}$ and cosolvent $\log P_{\mathrm{o}}^{\mathrm{COS}}$ (both measured), which indicates that acids and bases, when corrected for the $\mathrm{pH}$ effect (i.e., intrinsic values are compared), behave differently. This is reasonable, since the acetonitrile is a $\mathrm{H}$-bond acceptor, and the acid solutes are $\mathrm{H}$-bond donors, whereas the base solutes are H-bond acceptors.

Sugano and coworkers ${ }^{6}$ ) studied the effect of DMSO, PEG400, and ethanol, up to $30 \%$, in their PAMPA assays, but did not convert their results into aqueous (cosolvent-free) in- trinsic permeability values. In their regular assays, $5 \% \mathrm{v} / \mathrm{v}$ DMSO was present in both donor and acceptor wells. (Our solutions had $0.5 \%$ DMSO or less in the donor wells.) In general, water-miscible cosolvents are expected to decrease the membrane-water partition coefficients. Additionally, the decreased dielectric constants of the cosolvent-water solutions should give rise to a higher proportion of the ionizable molecule in the uncharged state. ${ }^{6,19)}$ These two effects oppose each other. Mostly, increasing levels of cosolvents were observed to lead to decreasing permeability in the Sugano study. However, ethanol made the weak acid ketoprofen more permeable with increasing cosolvent levels, an effect consistent with the increasing $\mathrm{p} K_{\mathrm{a}}$ with the decreasing dielectric constant of the cosolvent mixtures, which leads to a higher proportion of uncharged species at a given $\mathrm{pH}$. However, the weak base propranolol decreased in permeability with increasing amounts of ethanol. This may be due to the increased solubility of propranolol in water with the added ethanol in relation to the solubility in the membrane phase. The result is a lowered membrane/mixed-solvent partition coefficient lowering the flux due to the diminished sample 


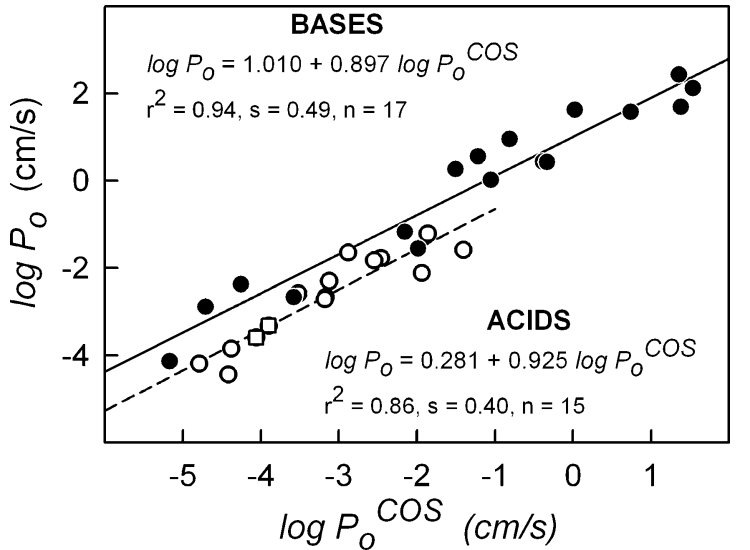

Fig. 2. Comparison of Intrinsic Permeability Coefficients Determined in Aqueous Buffer and in 20\% v/v Acetonitrile Buffer Solutions

The filled circles are bases, the open circles are acids, and the open squares are ampholytes.

concentration gradient in the membrane. ${ }^{19)}$

With the exception of gemfibrozil, ibuprofen, and sulphasalazine (Table 1), we also observed aqueous permeability coefficients to be greater than those based on cosolvent measurement. However, it must be emphasized that we used intrinsic permeability coefficients, which are already corrected for $\mathrm{pH}$ dependency, and we intentionally used aqueous $\mathrm{p} K_{\mathrm{a}}$ values when deriving $\log P_{\mathrm{o}}^{\mathrm{COS}}$. It is our experience that this strategy yields partial correction for the cosolvent effect on the permeability. When we tried to use $\mathrm{p} K_{\mathrm{a}}$ values measured in $20 \% \mathrm{v} / \mathrm{v}$ acetonitrile solution, the resulting $\log P_{\mathrm{o}}^{\mathrm{COS}}$ were lower by about one unit (data not shown). In our adopted approach, $\log P_{\mathrm{o}}-\log P_{\mathrm{o}}^{\mathrm{COS}}$ differences averaged to +0.52 for acids ( -0.18 to 1.23 range), and to +1.08 for bases ( 0.31 to 1.88 range).

The Unified Acid-Base in Silico Permeability Model The dependence of the intrinsic permeability coefficients of acids and bases on the properties of the two solvent systems can be described by distinct Collander-type ${ }^{24)}$ linear relationships, as Fig. 2 shows. To rationalize the class-specific differences, we used the Algorithm Builder computer program to find the most suitable descriptors, in addition to $\log P_{\mathrm{o}} \cos$, which would result in a unified acid-base model. Several combinations of solvation descriptors were tried: Abraham's ${ }^{21)} \mathrm{H}$-bond acidity $(\alpha)$ and basicity $(\beta)$, molecular volumes, polar surface area, and $\log P_{\text {oct }}$. The best model is described by the equation,

$$
\log P_{\mathrm{o}}=0.738+0.885 \log P_{\mathrm{o}}^{\mathrm{Cos}}-1.262 \alpha+0.436 \beta
$$

with the statistics $r^{2}=0.97, s=0.38, n=32$, and $F=279$. By including molecular volume as an additional descriptor, the $F$ value decreased to 211 , while the other statistics remain essentially unchanged. The $\beta$ and molecular volume had nearly equal contribution (suggesting strong correlation), while the other terms were unchanged. Neither the use of $\log P_{\text {oct }}$ nor polar surface area produced improvements in the intrinsic permeability model.

On the average, acids are stronger than bases as hydrogen bond donors, and conversely, bases are stronger than acids as hydrogen bond acceptors (Table 1). The substitution of the average $\alpha$ and $\beta$ values for acids and bases from Table 1 into Eq. 4 produces the class-specific regression equations listed

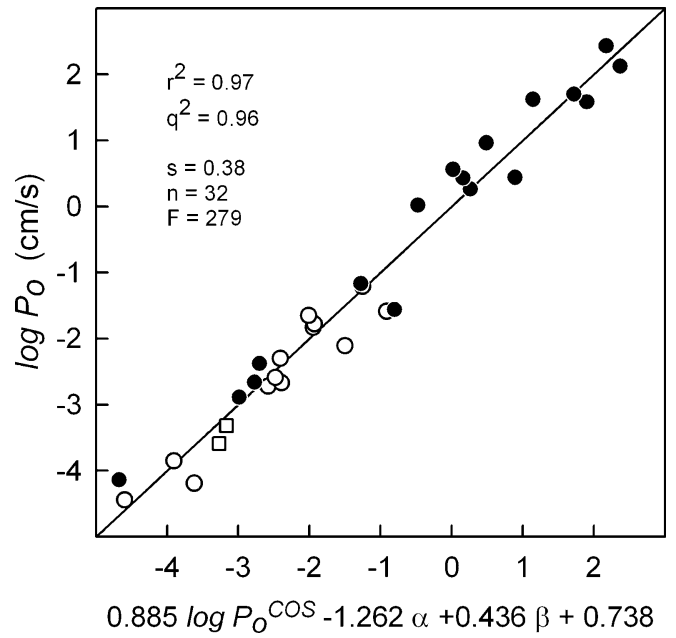

Fig. 3. The Unified Acid-Base Permeability Prediction Model, Using Abraham's H-Bond Descriptors

in Fig. 1. But why is the are there two classes to begin with?

In the mixed-solvent system, compared to the water system, membrane permeability of ionizable compounds should increase due to the lowered dielectric constant (higher fraction of uncharged species) and decrease due to the lowered membrane-buffer partition coefficient (increased solubility). These two effects oppose each other. The fact that acids are affected by the cosolvent less than bases (i.e., $\log P_{\mathrm{o}}-\log P_{\mathrm{o}}^{\cos }$ values are smaller for acids than bases), suggests the dominance of the dielectric effect. It is well established $^{19)}$ that water-miscible organic solvents cause the $\mathrm{p} K_{\mathrm{a}}$ values of acids to change a lot more than those of bases (leading to higher neutral-species fractions for acids). There appears to be less of a differentiation between acids and bases due to solvation enhancement of acetonitrile.

Figure 3 shows the results of the unified acid-base model. Table 1 summarizes the application of the unified acid-base model (Eq. 4) to molecules (application set) which could not be directly studied in aqueous buffer: amiodarone, itraconazole, miconazole, tamoxifen, and terfenadine. The corresponding aqueous intrinsic permeability coefficients are estimated as $13,3.2,0.32,28$, and $162 \mathrm{~cm} / \mathrm{s}$, respectively.

Model Validation The multiple linear regression (MLR) model developed in this study was validated by two variants of the leave-one-out (LOO) method. The traditional LOO approach, with 14 iterations of the MLR calculation, each time randomly taking out one measured permeability, produced the $q^{2}=0.96$. The leave-many-out (LMO) approach, where $20 \%$ of the dependent variables were removed, with the MLR repeated 14 times, produced the same $q^{2}=0.96$, with the $q^{2}$ standard deviation of 0.02 .

\section{Conclusion}

We conclude that the new UV-based cosolvent PAMPA method can be used to estimate the aqueous permeability of molecules as poorly soluble as $0.006 \mu \mathrm{g} / \mathrm{ml}$ (amiodarone), without compromising on the speed or cost of the assay. This is a particularly important advance in PAMPA, since so many of the test compounds in today's combinatorially-inspired discovery programs are so sparingly-soluble in water. 


\section{References and Notes}

1) Part 12 in the series: PAMPA - a Drug Absorption in Vitro Model. Ref. 15 is part 7.

2) Kansy M., Senner F., Gubernator K., J. Med. Chem., 41, 1007-1010 (1998).

3) Avdeef A., Strafford M., Block E., Balogh M. P., Chambliss W., Khan I., Eur. J. Pharm. Sci., 14, 271-280 (2001).

4) Wohnsland F., Faller B., J. Med. Chem., 44, 923-930 (2001).

5) Sugano K., Hamada H., Machida M., Ushio H., Saitoh K., Terada K., Int. J. Pharmaceut., 228, 181-188 (2001).

6) Sugano K., Hamada H., Machida M., Ushio H., J. Biomol. Screen., 6, $189-196$ (2001).

7) Sugano K., Takata N., Machida M., Saitoh K., Terada K., Int. J. Pharmaceut., 241, 241-251 (2002).

8) Sugano K., Nabuchi, Y., Machida M., Aso Y., Int. J. Pharmaceut., 257, $245-251$ (2003).

9) Sugano K., Nabuchi Y., Aso Y., Poster presented at the Academy of Pharmaceutical Sciences and Technolology (APSTJ) Meeting, April, Kyoto, Japan, April 2003.

10) Veber D. F., Johnson S. R., Cheng H.-Y., Smith B. R., Ward K. W., Kopple K. D., J. Med. Chem., 45, 2615-2623 (2002)

11) Zhu C., Jiang L., Chen T.-M., Hwang K.-K., Eur. J. Med. Chem., 37, 399-407 (2002).

12) Di L., Kerns E. H., Fan K., McConnell O. J., Carter G. T., Eur. J. Med.
Chem., 38, 223-232 (2003).

13) Liu H., Sabus C., Xu S., Carter G. T., Tischler M., Du C., Avdeef A., Pharm. Res., 20, 1820-1826 (2003).

14) Ruell J. A., Tsinman K. L., Avdeef A., Eur. J. Pharm. Sci., 20, 393 402 (2003).

15) Bermejo M., Avdeef A., Ruiz A., Nalda R., Ruell J. A., Tsinman O., Gonzalez I., Fernandez C., Sanchez G., Garrigues T. M., Merino V., Eur. J. Pharm. Sci., 21, 429-441 (2004).

16) Lipinski C. A., Based on a lecture presented at the Annual Meeting of the Society of Biomolecular Screening, The Hauge, The Netherlands on 24 Sept. 2002.

17) Di L., Kerns E. H., Curr. Opin. Chem. Biol., 7, $402-408$ (2003).

18) Kerns E. H., Di L., Curr. Top. Med. Chem., 2, 87-98 (2002).

19) Avdeef A., "Absorption and Drug Development," Wiley-Interscience, New York, 2003.

20) Kansy M., Fischer H., Kratzat K., Senner F., Wagner B., Parrilla I., "Pharmacokinetic Optimization in Drug Research," ed. by Testa B., van de Waterbeemd H., Folkers G., Guy R., Verlag Helvetica Chimica Acta, Zurich, 2001, pp. 447-464.

21) Abraham M. H., Chem. Soc. Revs., 22, 73-83 (1993).

22) Gutknecht J., Tosteson F. C., Science, 182, 1258-1261 (1973).

23) Walter A., Gutknecht J., J. Mem. Biol., 77, 255-264 (1984).

24) Collander R., Acta Chem. Scand., 5, 774-780 (1951). 